\title{
GBS operators of Durrmeyer-Stancu type
}

\author{
Ovidiu T. Pop and Dan Bărbosu
}




\title{
GBS OPERATORS OF DURRMEYER-STANCU TYPE
}

\author{
OVIDIU T. POP AND DAN BĂRBOSU
}

Received 4 July, 2005

\begin{abstract}
Considering four given real parameters $\alpha_{1}, \beta_{1}, \alpha_{2}$, and $\beta_{2}$, which satisfy the conditions $0 \leq \alpha_{1} \leq \beta_{1}$ and $0 \leq \alpha_{2} \leq \beta_{2}$, we construct the bivariate Durrmeyer-Stancu operators $D_{m, n}^{\left(\alpha_{1}, \beta_{1}\right)\left(\alpha_{2}, \beta_{2}\right)}$ defined by relation (3.1). Next, we consider the associated GBS (Generalized Boolean Sum) operators and establish some approximation properties of these operators.

2000 Mathematics Subject Classification: 41A25, 41A36, 41A63

Keywords: linear positive operators, Durrmeyer operators, Stancu operators, bivariate operators, mixed modulus of smoothness, approximation of functions by GBS operators
\end{abstract}

\section{INTRODUCTION}

In 1967, J. L. Durrmeyer [3] introduced the operators $D_{m}: L_{1}([0,1]) \rightarrow C([0,1])$ defined by the formula

$$
\left(D_{m} f\right)(x)=(m+1) \sum_{k=0}^{m} p_{m, k}(x) \int_{0}^{1} p_{m, k}(t) f(t) d t,
$$

for any $f \in L_{1}([0,1])$, any $x \in[0,1]$, and any positive integer $m$, where

$$
p_{m, k}(x)=\left(\begin{array}{c}
m \\
k
\end{array}\right) x^{k}(1-x)^{m-k}, \quad x \in[0,1]
$$

are the fundamental Bernstein polynomials. These operators are called the Durrmeyer operators.

Let $\alpha$ and $\beta$ be two given real parameters satisfying the conditions $0 \leq \alpha \leq \beta$. In the paper [5], D. D. Stancu introduced and studied the linear positive operators $P_{m}^{(\alpha, \beta)}: C([0,1]) \rightarrow C([0,1])$ defined for any function $f \in C([0,1])$ and any positive integer $m$ by the formula

$$
\left(P_{m}^{(\alpha, \beta)} f\right)(x)=\sum_{k=0}^{m} p_{m, k}(x) f\left(\frac{k+\alpha}{m+\beta}\right)
$$

for all $x \in[0,1]$. These operators are called the Stancu operators. 
In the paper [4], the authors introduced and studied the Durrmeyer-Stancu type operators $\left(D_{m}^{(\alpha, \beta)}\right)_{m \geq 1}$ for two given real parameters $\alpha$ and $\beta$ with $0 \leq \alpha \leq \beta$. The linear operators $D_{m}^{(\alpha, \beta)}: L_{1}([0,1]) \rightarrow C([0,1])$ are defined, for any function $f \in$ $L_{1}([0,1])$ and any positive integer $m$, by the relation

$$
\left(D_{m}^{(\alpha, \beta)} f\right)(x)=(m+1) \sum_{k=0}^{m} p_{m, k}(x) \int_{0}^{1} p_{m, k}(t) f\left(\frac{m t+\alpha}{m+\beta}\right) d t
$$

for any $x \in[0,1]$.

\section{Preliminaries}

In the following, we recall some results which we will use in this paper (see [1,2, 4]). Let the real numbers $a, b, c, d$ so that $a<b, c<d$,

$$
C_{b}([a, b] \times[c, d])=\{f:[a, b] \times[c, d] \rightarrow \mathbb{R}: f B \text {-continuous on }[a, b] \times[c, d]\},
$$

$M([a, b] \times[c, d])=\{f:[a, b] \times[c, d] \rightarrow \mathbb{R}: f$ bounded on $[a, b] \times[c, d]\}$,

and $e_{i j}(x, y)=x^{i} y^{j}, i, j \in \mathbb{N}, 0 \leq i, j \leq 2$ are the test functions.

If $f:[a, b] \times[c, d] \rightarrow \mathbb{R}, f$ is $B$-bounded function on $[a, b] \times[c, d]$, then the function $\omega_{\text {mixed }}:[0, b-a] \times[0, d-c] \rightarrow \mathbb{R}$, defined by the formula

$$
\omega_{\text {mixed }}\left(f ; \delta_{1}, \delta_{2}\right)=\sup \left\{|(\Delta f)((x, y),(s, t))|:|s-x| \leq \delta_{1},|t-y| \leq \delta_{2}\right\}
$$

for any $\left(\delta_{1}, \delta_{2}\right) \in[0, b-a] \times[0, d-c]$, is called the mixed modulus of smoothness of function $f$.

Theorem 2.1. Let $\left(L_{m, n}\right)_{m, n \in \mathbb{N}}$ be a sequence of linear positive bivariate operators, $L_{m, n}: C_{b}([a, b] \times[c, d]) \rightarrow M([a, b] \times[c, d]), m, n \in \mathbb{N}$. If

(i) $\left(L_{m, n} e_{00}\right)(x, y)=1$,

(ii) $\left(L_{m, n} e_{10}\right)(x, y)=e_{10}(x, y)+u_{m, n}(x, y)$,

(iii) $\left(L_{m, n} e_{01}\right)(x, y)=e_{01}(x, y)+v_{m, n}(x, y)$,

(iv) $\left(L_{m, n} e_{22}\right)(x, y)=e_{22}(x, y)+w_{m, n}(x, y)$,

(v) $\lim _{m, n \rightarrow \infty} u_{m, n}(x, y)=\lim _{m, n \rightarrow \infty} v_{m, n}(x, y)=\lim _{m, n \rightarrow \infty} w_{m, n}(x, y)=$ 0 uniformly on the rectangle $[a, b] \times[c, d]$,

then, for any $f \in C_{b}([a, b] \times[c, d])$, the sequence $\left(U_{m, n} f\right)_{m, n \in \mathbb{N}}$ converges uniformly to $f$ on the rectangle $[a, b] \times[c, d]$, where, for any $m, n \in \mathbb{N}$, the operator $U_{m, n}: \mathbb{R}^{[a, b] \times[c, d]} \rightarrow \mathbb{R}^{[a, b] \times[c, d]}$ is defined by

$$
\left(U_{m, n} f\right)(x, y)=\left(L_{m, n}(f(\cdot, y)+f(x, *)-f(\cdot, *))\right)(x, y)
$$

for any $f \in \mathbb{R}^{[a, b] \times[c, d]}$, any $(x, y) \in[a, b] \times[c, d]$, “." and “*” stand for the first and second variable. 
Remark 2.1. For the positive integers $m$ and $n$, the operator $U_{m, n}$ is called GBS operator associated to $L_{m, n}$ operator. The term "GBS operator" (Generalized Boolean Sum operator) was introduced by C. Badea and C. Cottin in the paper [2].

Remark 2.2. Theorem 2.1 is a Korovkin type theorem for $B$-continuous functions and is due to C. Badea, I. Badea, and H. H. Gonska (see [1]).

Theorem 2.2. Let $\left(L_{m, n}\right)_{m, n \in \mathbb{N}}$ be an arbitrary sequence of linear positive operators $L_{m, n}: C_{b}([a, b] \times[c, d]) \rightarrow M([a, b] \times[c, d])$ and $\left(U_{m, n}\right)_{m, n \in \mathbb{N}}$ the sequence of associated GBS operators. Then, for any $f \in C_{b}([a, b] \times[c, d])$, any $(x, y) \in$ $[a, b] \times[c, d]$, any $\left(\delta_{1}, \delta_{2}\right) \in[0, b-a] \times[0, d-c]$ and any positive integers $m, n$, the following

$$
\begin{aligned}
\mid\left(U_{m, n} f\right) & (x, y)-f(x, y)|\leq| f(x, y)|| 1-\left(L_{m, n} e_{00}\right)(x, y) \mid \\
& +\left(\left(L_{m, n} e_{00}\right)(x, y)+\delta_{1}^{-1} \sqrt{\left(L_{m, n}(\cdot-x)^{2}\right)(x, y)}\right. \\
& +\delta_{2}^{-1} \sqrt{\left(L_{m, n}(*-y)^{2}\right)(x, y)} \\
& \left.+\delta_{1}^{-1} \delta_{2}^{-1} \sqrt{\left(L_{m, n}(\cdot-x)^{2}(*-y)^{2}\right)(x, y)}\right) \omega_{\text {mixed }}\left(f ; \delta_{1}, \delta_{2}\right)
\end{aligned}
$$

holds.

Remark 2.3. Theorem 2.2 is a Shisha-Mond type theorem for $B$-continuous functions; it is due to C. Badea and C. Cottin (see [2]).

Theorem 2.3. Let two given real parameters $\alpha$ and $\beta$ with $0 \leq \alpha \leq \beta$. The oprators $\left(D_{m}^{(\alpha, \beta)}\right)_{m \geq 1}$ verify the following

$$
\begin{aligned}
&\left(D_{m}^{(\alpha, \beta)} e_{0}\right)(x)=1, \\
&\left(D_{m}^{(\alpha, \beta)} e_{1}\right)(x)= \frac{m^{2}}{(m+\beta)(m+2)} x+\frac{(\alpha+1) m+2 \alpha}{(m+\beta)(m+2)}, \\
&\left(D_{m}^{(\alpha, \beta)} e_{2}\right)(x)= \frac{m^{3}(m-1)}{(m+\beta)^{2}(m+2)(m+3)} x^{2} \\
&+\frac{4 m^{3}+2 \alpha m^{2}(m+3)}{(m+\beta)^{2}(m+2)(m+3)} x \\
&+\frac{2 m^{2}+2 \alpha m(m+3)+\alpha^{2}(m+2)(m+3)}{(m+\beta)^{2}(m+2)(m+3)}, \\
&\left(D_{m}^{(\alpha, \beta)} \varphi_{x}^{2}\right)(x)=\left(\frac{m}{m+\beta}\right)^{2} \frac{2(m-3) x(1-x)+2}{(m+2)(m+3)} {\left[\beta^{2}(m+2)+4 \beta m\right] x^{2}-2 x[\alpha \beta(m+2)+\beta m+2 \alpha m] x+\alpha^{2}(m+2)+2 \alpha m } \\
&+\frac{(m+\beta)^{2}(m+2)}{(m+2)}
\end{aligned}
$$


and

$$
\delta_{m}^{(\alpha, \beta)}(x) \leq \delta_{m}^{(\alpha, \beta)}
$$

where

$$
\delta_{m}^{(\alpha, \beta)}(x)=\sqrt{\left(D_{m}^{(\alpha, \beta)} \varphi_{x}^{2}\right)(x)}
$$

and

$$
\begin{aligned}
\delta_{m}^{(\alpha, \beta)}= & \left(\left(\frac{m}{m+\beta}\right)^{2} \frac{m+1}{2(m+2)(m+3)}\right. \\
& \left.+\max \left\{\frac{\alpha^{2}(m+2)+2 \alpha m}{(m+\beta)^{2}(m+2)}, \frac{(\alpha-\beta)^{2}(m+2)-2(\alpha-\beta) m}{(m+\beta)^{2}(m+2)}\right\}\right)^{\frac{1}{2}}
\end{aligned}
$$

for any positive integer $m$, and any $x \in[0,1]$.

Proof. For the proof, see [4].

\section{MAIN RESULTS}

In this section, let the given real parameters $\alpha_{1}, \alpha_{2}, \beta_{1}$, and $\beta_{2}$ satisfying the conditions $0 \leq \alpha_{1} \leq \beta_{1}$ and $0 \leq \alpha_{2} \leq \beta_{2}$.

We construct the bivariate Durrmeyer-Stancu operators

$$
D_{m, n}^{\left(\alpha_{1}, \beta_{1}\right)\left(\alpha_{2}, \beta_{2}\right)}: L_{1}([0,1] \times[0,1]) \rightarrow C([0,1] \times[0,1])
$$

defined for any positive integers $m, n$ and any $f \in L_{1}([0,1] \times[0,1])$ by

$$
\begin{aligned}
\left(D_{m, n}^{\left(\alpha_{1}, \beta_{1}\right)\left(\alpha_{2}, \beta_{2}\right)}\right. & f)(x, y)=(m+1)(n+1) \sum_{k=0}^{m} \sum_{j=0}^{n} p_{m, k}(x) p_{n, j}(y) \\
& \times \int_{0}^{1} \int_{0}^{1} p_{m, k}(s) p_{n, j}(t) f\left(\frac{m s+\alpha_{1}}{m+\beta_{1}}, \frac{n t+\alpha_{2}}{n+\beta_{2}}\right) d s d t
\end{aligned}
$$

for any $(x, y) \in[0,1] \times[0,1]$.

Let ${ }^{x} D_{m}^{\left(\alpha_{1}, \beta_{1}\right)},{ }^{y} D_{n}^{\left(\alpha_{2}, \beta_{2}\right)}$ be the parametric extensions of the operator (1.3), where $x, y \in[0,1]$.

If $x \in[0,1]$, then ${ }^{x} D_{m}^{\left(\alpha_{1}, \beta_{1}\right)}: L_{1}([0,1] \times[0,1]) \rightarrow C([0,1] \times[0,1])$ is defined for any positive integer $m$ and any $f \in L_{1}([0,1] \times[0,1])$ by the formula

$$
\left({ }^{x} D_{m}^{\left(\alpha_{1}, \beta_{1}\right)} f\right)(x, y)=(m+1) \sum_{k=0}^{m} p_{m, k}(x) \int_{0}^{1} p_{m, k}(s) f\left(\frac{m s+\alpha_{1}}{m+\beta_{1}}, y\right) d s
$$


for any $y \in[0,1]$ and if $y \in[0,1]$, then ${ }^{y} D_{n}^{\left(\alpha_{2}, \beta_{2}\right)}: L_{1}([0,1] \times[0,1]) \rightarrow C([0,1] \times[0,1])$ is defined for any positive integer $n$ and any $f \in L_{1}([0,1] \times[0,1])$ by the formula

$$
\left({ }^{y} D_{n}^{\left(\alpha_{1}, \beta_{2}\right)} f\right)(x, y)=(n+1) \sum_{j=0}^{n} p_{n, j}(y) \int_{0}^{1} p_{n, j}(t) f\left(x, \frac{n t+\alpha_{2}}{n+\beta_{2}}\right) d t
$$

for any $x \in[0,1]$.

The GBS operator of Durrmeyer-Stancu is the "boolean sum" of the parametric extensions of the operators ${ }^{x} D_{m}^{\left(\alpha_{1}, \beta_{1}\right)}$ and ${ }^{y} D_{n}^{\left(\alpha_{2}, \beta_{2}\right)}$, so

$$
U_{m, n}^{\left(\alpha_{1}, \beta_{1}\right)\left(\alpha_{2}, \beta_{2}\right)}: L_{1}([0,1] \times[0,1]) \rightarrow C([0,1] \times[0,1])
$$

is defined for any positive integers $m$ and $n$ by the relation

$$
\begin{aligned}
U_{m, n}^{\left(\alpha_{1}, \beta_{1}\right)\left(\alpha_{2}, \beta_{2}\right)} & ={ }^{x} D_{m}^{\left(\alpha_{1}, \beta_{1}\right)} \oplus{ }^{y} D_{n}^{\left(\alpha_{2}, \beta_{2}\right)}= \\
& ={ }^{x} D_{m}^{\left(\alpha_{1}, \beta_{1}\right)}+{ }^{y} D_{n}^{\left(\alpha_{2}, \beta_{2}\right)}-{ }^{x} D_{m}^{\left(\alpha_{1}, \beta_{1}\right)} \circ{ }^{y} D_{n}^{\left(\alpha_{2}, \beta_{2}\right)},
\end{aligned}
$$

where

$$
{ }^{x} D_{m}^{\left(\alpha_{1}, \beta_{1}\right)} \circ{ }^{y} D_{n}^{\left(\alpha_{2}, \beta_{2}\right)}=D_{m, n}^{\left(\alpha_{1}, \beta_{1}\right)\left(\alpha_{2}, \beta_{2}\right)} .
$$

Lemma 3.1. The Durrmeyer-Stancu type GBS operator is defined, for any $f \in$ $L_{1}([0,1] \times[0,1])$ and any positive integers $m$ and $n$, by the relation

$$
\begin{aligned}
& \left(U_{m, n}^{\left(\alpha_{1}, \beta_{1}\right)\left(\alpha_{2}, \beta_{2}\right)} f\right)(x, y)=(m+1)(n+1) \sum_{k=0}^{m} \sum_{j=0}^{n} p_{m, k}(x) p_{n, j}(y) \\
& \quad \times \int_{0}^{1} \int_{0}^{1} p_{m, k}(s) p_{n, j}(t)\left(f\left(\frac{m s+\alpha_{1}}{m+\beta_{1}}, y\right)+f\left(x, \frac{n t+\alpha_{2}}{n+\beta_{2}}\right)\right. \\
& \left.\quad-f\left(\frac{m s+\alpha_{1}}{m+\beta_{1}}, \frac{n t+\alpha_{2}}{n+\beta_{2}}\right)\right) d s d t
\end{aligned}
$$

for all $(x, y) \in[0,1] \times[0,1]$.

Proof. The assertion follows from definitions (3.1)-(3.4).

Lemma 3.2. For any positive integers $m, n$ and any $(x, y) \in[0,1] \times[0,1]$, the parametric extensions ${ }^{x} D_{m}^{\left(\alpha_{1}, \beta_{1}\right)}$ and ${ }^{y} D_{n}^{\left(\alpha_{2}, \beta_{2}\right)}$ are linear and positive operators.

Proof. The assertion is obtained by direct computation.

Lemma 3.3. operators Let $m$ and $n$ be given positive integers. The operator $D_{m, n}^{\left(\alpha_{1}, \beta_{1}\right)\left(\alpha_{2}, \beta_{1}\right)}$ is linear and positive.

Proof. The proof is immediate.

The operator $D_{m, n}^{\left(\alpha_{1}, \beta_{1}\right)\left(\alpha_{2}, \beta_{1}\right)}$ is called in what follows the bivariate DurrmeyerStancu operator. 
Lemma 3.4. For any nonzero natural numbers $m, n$ and arbitrary $(x, y) \in[0,1] \times$ $[0,1]$, we have

$$
\begin{aligned}
& \left(D_{m, n}^{\left(\alpha_{1}, \beta_{1}\right)\left(\alpha_{2}, \beta_{2}\right)} e_{00}\right)(x, y)=1 \\
& \left(D_{m, n}^{\left(\alpha_{1}, \beta_{1}\right)\left(\alpha_{2}, \beta_{2}\right)} e_{10}\right)(x, y)=\frac{m^{2}}{\left(m+\beta_{1}\right)(m+2)} x+\frac{\left(\alpha_{1}+1\right) m+2 \alpha_{1}}{\left(m+\beta_{1}\right)(m+2)}, \\
& \left(D_{m, n}^{\left(\alpha_{1}, \beta_{1}\right)\left(\alpha_{2}, \beta_{2}\right)} e_{01}\right)(x, y)=\frac{n^{2}}{\left(n+\beta_{2}\right)(n+2)} y+\frac{\left(\alpha_{2}+1\right) n+2 \alpha_{2}}{\left(n+\beta_{2}\right)(n+2)} \\
& \left(D_{m, n}^{\left(\alpha_{1}, \beta_{1}\right)\left(\alpha_{2}, \beta_{2}\right)} e_{22}\right)(x, y)=\frac{m^{3}(m-1)}{\left(m+\beta_{1}\right)^{2}(m+2)(m+3)} x^{2} \\
& +\frac{4 m^{3}+2 \alpha_{1} m^{2}(m+3)}{\left(m+\beta_{1}\right)^{2}(m+2)(m+3)} x+\frac{2 m^{2}+2 \alpha_{1} m(m+3)+\alpha_{1}^{2}(m+2)(m+3)}{\left(m+\beta_{1}\right)^{2}(m+2)(m+3)} \\
& +\frac{n^{3}(n-1)}{\left(n+\beta_{2}\right)^{2}(n+2)(n+3)} y^{2} \frac{4 n^{3}+2 \alpha_{2} n^{2}(n+3)}{\left(n+\beta_{2}\right)^{2}(n+2)(n+3)} y \\
& +\frac{2 n^{2}+2 \alpha_{2} n(n+3)+\alpha_{2}^{2}(n+2)(n+3)}{\left(n+\beta_{2}\right)^{2}(n+2)(n+3)}, \\
& \left(D_{m, n}^{\left(\alpha_{1}, \beta_{1}\right)\left(\alpha_{2}, \beta_{2}\right)}(\cdot-x)^{2}\right)(x, y)=\left(D_{m}^{\left(\alpha_{1}, \beta_{1}\right)} \varphi_{x}^{2}\right)(x)=\left(\delta_{m}^{\left(\alpha_{1}, \beta_{1}\right)}(x)\right)^{2}, \\
& \left(D_{m, n}^{\left(\alpha_{1}, \beta_{1}\right)\left(\alpha_{2}, \beta_{2}\right)}(*-y)^{2}\right)(x, y)=\left(D_{n}^{\left(\alpha_{2}, \beta_{2}\right)} \varphi_{y}^{2}\right)(y)=\left(\delta_{n}^{\left(\alpha_{2}, \beta_{2}\right)}(y)\right)^{2} \\
& \quad \delta_{m}^{\left(\alpha_{1}, \beta_{1}\right)}(x) \leq \delta_{m}^{\left(\alpha_{1}, \beta_{1}\right)}
\end{aligned}
$$

and

$$
\delta_{n}^{\left(\alpha_{2}, \beta_{2}\right)}(y) \leq \delta_{n}^{\left(\alpha_{2}, \beta_{2}\right)},
$$

where the values $\delta_{m}^{\left(\alpha_{1}, \beta_{1}\right)}(x), \delta_{m}^{\left(\alpha_{1}, \beta_{1}\right)}, \delta_{n}^{\left(\alpha_{2}, \beta_{2}\right)}(y)$, and $\delta_{n}^{\left(\alpha_{2}, \beta_{2}\right)}$ are defined by relations (2.7)-(2.9).

Proof. The assertion follows from Theorem 2.3.

Lemma 3.5. The bivariate Durrmeyer-Stancu operators satisfy the relations

$$
\begin{aligned}
& \lim _{m, n \rightarrow \infty}\left(D_{m, n}^{\left(\alpha_{1}, \beta_{1}\right)\left(\alpha_{2}, \beta_{2}\right)} e_{10}\right)(x, y)=e_{10}(x, y), \\
& \lim _{m, n \rightarrow \infty}\left(D_{m, n}^{\left(\alpha_{1}, \beta_{1}\right)\left(\alpha_{2}, \beta_{2}\right)} e_{01}\right)(x, y)=e_{01}(x, y),
\end{aligned}
$$

and

$$
\lim _{m, n \rightarrow \infty}\left(D_{m, n}^{\left(\alpha_{1}, \beta_{1}\right)\left(\alpha_{2}, \beta_{2}\right)} e_{22}\right)(x, y)=e_{22}(x, y)
$$

uniformly on $[0,1] \times[0,1]$. 
Proof. Relations (3.14), (3.15), and (3.16) are consequences of Lemma 3.4.

Theorem 3.1. For any $f \in C_{b}([0,1] \times[0,1]) \cap L_{1}([0,1] \times[0,1])$, the sequence

$$
\left(U_{m, n}^{\left(\alpha_{1}, \beta_{1}\right)\left(\alpha_{2}, \beta_{2}\right)} f\right)_{m, n \geq 1}
$$

converges to $f$ uniformly on $[0,1] \times[0,1]$.

Proof. It is sufficient to apply Theorem 2.1 and Lemma 3.5.

Theorem 3.2. For any positive integers $m$ and $n$, any function $f \in C_{b}([0,1] \times$ $[0,1]) \cap L_{1}([0,1] \times[0,1])$ and any $(x, y) \in[0,1] \times[0,1]$, the operator $U_{m, n}^{\left(\alpha_{1}, \beta_{1}\right)\left(\alpha_{2}, \beta_{2}\right)}$ has the properties

$$
\begin{aligned}
& \left|\left(U_{m, n}{ }^{\left(\alpha_{1}, \beta_{1}\right)\left(\alpha_{2}, \beta_{2}\right)} f\right)(x, y)-f(x, y)\right| \leq\left(1+\frac{1}{\delta_{1}} \delta_{m}^{\left(\alpha_{1}, \beta_{1}\right)}(x)\right. \\
& \left.\quad+\frac{1}{\delta_{2}} \delta_{n}^{\left(\alpha_{2}, \beta_{2}\right)}(y)+\frac{1}{\delta_{1} \delta_{2}} \delta_{m}^{\left(\alpha_{1}, \beta_{1}\right)}(x) \delta_{n}^{\left(\alpha_{2}, \beta_{2}\right)}(y)\right) \omega_{\text {mixed }}\left(f ; \delta_{1}, \delta_{2}\right)
\end{aligned}
$$

for any $\delta_{1}, \delta_{2} \in(0,1]$ and

$$
\begin{aligned}
\mid\left(U_{m, n}^{\left(\alpha_{1}, \beta_{1}\right)\left(\alpha_{2}, \beta_{2}\right)} f\right)(x, y) & -f(x, y) \mid \\
& \leq 4 \omega_{\text {mixed }}\left(f ; \delta_{m}^{\left(\alpha_{1}, \beta_{1}\right)}(x), \delta_{n}^{\left(\alpha_{2}, \beta_{2}\right)}(y)\right) \\
& \leq 4 \omega_{\text {mixed }}\left(f ; \delta_{m}^{\left(\alpha_{1}, \beta_{1}\right)}, \delta_{n}^{\left(\alpha_{2}, \beta_{2}\right)}\right) .
\end{aligned}
$$

Proof. For the first inequality (3.17), we apply Theorem 2.2, Lemma 3.4, and relations (3.10) and (3.11). For the inequality (3.18), in the inequality (3.17) we choose

$$
\begin{aligned}
& \delta_{1}=\delta_{m}^{\left(\alpha_{1}, \beta_{1}\right)}(x), \\
& \delta_{2}=\delta_{n}^{\left(\alpha_{2}, \beta_{2}\right)}(y),
\end{aligned}
$$

and afterwards we take the relations (3.12) and (3.13) into account.

\section{REFERENCES}

[1] C. Badea, I. Badea, and H. H. Gonska, "A test function theorem and approximation by pseudopolynomials," Bull. Austral. Math. Soc., vol. 34, no. 1, pp. 53-64, 1986.

[2] C. Badea and C. Cottin, "Korovkin-type theorems for generalized Boolean sum operators," in Approximation theory (Kecskemét, 1990), ser. Colloq. Math. Soc. János Bolyai. Amsterdam: NorthHolland, 1991, vol. 58, pp. 51-68.

[3] J. L. Durrmeyer, "Une formule d'inversion de la transformée de Laplace: Application à la theorie des moments," Thèse de 3e cycle, Faculté des Sciences de l'Université de Paris, 1967.

[4] O. T. Pop and D. Bărbosu, "Durrmeyer-Stancu type operators," to appear.

[5] D. D. Stancu, "On a generalization of the Bernštel̆n polynomials," Studia Univ. Babeş-Bolyai Ser. Math.-Phys., vol. 14, no. 2, pp. 31-45, 1969. 
Authors' addresses

Ovidiu T. Pop

Vest University "Vasile Goldiş" of Arad, Branch of Satu Mare, 26 Mihai Viteazu St., Satu Mare 440030, Romania

E-mail address: ovidiutiberiu@yahoo.com

Dan Bărbosu

North University of Baia Mare, Department of Mathematics and Computer Science, 76 Victoriei St., 43012 Baia Mare, Romania

E-mail address: danbarbosulyahoo.com 